\title{
Aerosol Optical Thickness Retrieval over Land from MODIS Data on Remote Sensing Information Service Grid Node
}

\author{
Jianping Guo ${ }^{1,3}$, Yong Xue ${ }^{1,2, *}$, Ying Wang ${ }^{1,3}$, Yincui $\mathrm{Hu}^{1,3}$, Jianqin Wang ${ }^{4}$, \\ Ying Luo ${ }^{1,3}$, Shaobo Zhong ${ }^{1,3}$, Wei Wan ${ }^{1,3}$, Lei Zheng ${ }^{1,3}$, and Guoyin Cai ${ }^{1,3}$ \\ ${ }^{1}$ State Key Laboratory of Remote Sensing Science, Jointly Sponsored by the Institute of \\ Remote Sensing Applications of Chinese Academy of Sciences and Beijing Normal University, \\ Institute of Remote Sensing Applications, Chinese Academy of Sciences, PO Box 9718, \\ Beijing 100101, China \\ ${ }^{2}$ Department of Computing, London Metropolitan University, 166-220 Holloway Road, \\ London N7 8DB, UK \\ ${ }^{3}$ Graduate University, Chinese Academy of Sciences, Beijing 100049, China \\ ${ }^{4}$ College of Information and Electrical Engineering, China Agricultural University, PO Box \\ 142, Beijing, 10083, China \\ jpguo_irsa@hotmail.com, y.xue@londonmet.ac.uk
}

\begin{abstract}
The signal at the top of the atmosphere will certainly contain information about both the surface and the atmosphere. To derive the geophysical parameters from satellites remote sensing images, the atmospheric effects must be decoupled. Aerosol Optical Thickness (AOT), an important aerosol optical property, should be correctly determined to remove the atmospheric effect. The retrieval process is great time-consuming and the EMS memory required is too large for a personal computing to run efficiently. Therefore, to facilitate the process smoothly, SYNTAM model is used to retrieve AOT over a wide range of land including China and one European area from MODIS data on the Remote Sensing Information Service Grid Node (RSIN, http://www.tgp.ac.cn) deployed at Institute of Remote Sensing Applications, Chinese Academy of Sciences. AOT retrieval results show that the RSIN Grid service is high efficient and has the potential to be applied to the remote sensing parameter inversion.
\end{abstract}

Keywords: Aerosol Optical Thickness Retrieval, MODIS Data, Remote Sensing Information Service Grid Node.

\section{Introduction}

Aerosols are important components of the atmosphere that influence the Earth's energy balance both directly (by scattering and absorbing radiation) and indirectly (by serving as nuclei for cloud formation), and affect hydrological cycle (IPCC, 2001).

\footnotetext{
* Corresponding author.
} 
They also affect public health and reduce visibility (Jacovides et al., 1994). Aerosol particles have heterogeneous spatial and temporal distributions with life spans on the order of days to weeks (IPCC 2001). Satellite observations of aerosol concentrations are thought to contribute greatly to reduce the large uncertainty in current estimates of aerosol-caused radiative forcing (Varotsos et al., 2006).

AOT can be retrieved from a wide range of satellites sensors including Moderate Resolution Imaging Spectroradiometer (MODIS), MISR (Multiangle Imaging SpectroRadiometer), AVHRR (Advanced Very High Resolution Radiometer), POLDER (Polarization and Directionary of the Earth's Reflectances). The operational retrieval of the AOT over sea suggests that the algorithm is matured. Derivation of AOT over land, however, remains great uncertainty due to its high variation of land type. DDV method has been proposed among researchers to retrieve aerosol properties over such dark targets as water bodies and vegetation areas (Liu et. al., 1996).

MODIS is a sensor with the ability to characterize the spatial and temporal characteristics of the global aerosol field. Launched aboard NASA's Terra and Aqua satellites in December 1999 and May 2002, MODIS has 36 channels spanning the spectral range from 0.41 to $15 \mu \mathrm{m}$ representing three spatial resolutions: $250 \mathrm{~m}, 500 \mathrm{~m}$, and $1 \mathrm{~km}$. The aerosol retrieval makes use of seven of these channels $(0.47-2.1 \mu \mathrm{m})$ to retrieve AOT and properties (Kaufman et al., 1997).

When assuming the aerosol optical property invariable and the temporal differences between the two satellite overpasses over the same region ignored, the Synergy of TERRA and AQUA MODIS data (SYNTAM) algorithm is used to retrieval AOT from MODIS (Tang et al. 2005).

Grid computing aggregates heterogeneous resources and provides hardware and software services, supporting application and services composition, workflow expression, scheduling, and execution management and service level agreements based allocation of resources. It has been an enabled environment for data sharing and processing.

Researchers and corporations have developed different types of grid computing platforms including support resource pooling or sharing such as SETI@Home, Condor, and Alchemi harnessing idle CPU cycles from desktop computers in the network, Globus, EU DataGrid, and Gridbus allow sharing of computational and distributed data resources. Guo et al., (2005) proposed a grid-based spatial epidemiology applicationa for scientists from both biological and spatial information fields. With respect to remote sensing applications based on grid service Aloisio et al. (2004) proposed a grid architecture, then after that, the grid platform of remote sensing data processing is developed (Aloisio et al. 2003).

In this paper we focus on the implementation of aerosol optical thickness retrieval on RSIN developed by Telegeoprocessing Research Group in Institute of Remote Sensing Applications (IRSA), Chinese Academy of Sciences (CAS), providing more than ten geophysical parameter retrieval functions (Luo et al., 2006). The remainder of this paper is organized as follows: in Section 2, the algorithm of AOT retrieval is introduced in detail. The architecture and process of the AOT retrieval service are presented in Section 3. Two AOT retrieval experiments over China and one European 
area are performed on RSIN. Finally, some conclusions are drawn about the AOT retrieval implementation by means of Grid service platform-RSIN, and future work is discussed.

\section{SYNTAM Model}

The Synergy of Terra and Aqua MODIS data (SYNTAM) algorithm is used to retrieval AOT in this paper. The aerosol retrieval model bases on Equation (1) (Xue and Cracknell 1995).

$$
A_{j, \lambda_{i}}=\frac{\left(A_{j, \lambda_{i}}{ }^{\prime} b-a_{j}\right)+a_{j}\left(1-A_{j, \lambda_{i}}\right) e^{\left(a_{j}-b\right) \varepsilon\left(0.00879_{i}^{-409}+\beta_{j} \lambda_{i}^{-\alpha}\right) \sec \theta_{j}^{\prime}}}{\left(A_{j, \lambda_{i}} b-a_{j}\right)+b\left(1-A_{j, \lambda_{i}}\right) e^{\left(a_{j}-b\right) \varepsilon\left(0.00879_{i}^{-4.09}+\beta_{j} \lambda_{i}^{-\alpha}\right) \sec \theta_{j}^{\prime}}} .
$$

where $j=1,2$, respectively stands for the observation of TERRA and AQUA; $i=1,2,3$, respectively stands for three visible spectral bands with central wavelength at $0.47 \mu \mathrm{m}$, $0.55 \mu \mathrm{m}, 0.66 \mu \mathrm{m} ; \lambda$ is the central wavelength. A is the Earth's surface reflectance. $\mathrm{A}^{\prime}$ is the Earth's system reflectance (Tang, et al.2005).

The SYNTAM algorithm infers the surface-leaving and path radiance contributions to total observed radiance without any assumption regarding the absolute surface reflectance and the land type, but with assumption regarding the invariance of wavelength exponent $\alpha$ during time intervals of Terra and Aqua satellites overpass time (10:30, 13:30, local time, respectively).

The process of AOT retrieval using SYNTAM includes fourfold as follows: first of all, the input images from both Terra and Aqua should be georeferenced before coregistration are performed. Secondly, the radiance calibration is performed to get the correct physical parameters including sensor zenith angle, solar zenith angle, and top of atmosphere reflectance. Thirdly, cloud should be screened and removed. Finally all pixel values, as input parameters, are to SYNTAM to derive AOT. The retrieval results may be post-processed and then provided to the end users through the RSIN Grid service.

\section{AOT Retrieval Services on RSIN}

\subsection{Remote Sensing Information Service Grid Node}

Remotely sensed data is one of the most important spatial information sources, so are the researches on architectures and technical supports of remote sensing information analysis.

RSIN, a significant part of the research on SIG, aims at integrating data, traditional algorithm and software, and computing resource distributed, provide one-stop service to everyone on Internet, and make good use of everything pre-existing. The node can be very large, which contains many personal computers, supercomputers or other 
nodes. It also can be as small as just on personal computer. Figure 1 describes the architecture of Remote sensing information analysis and service Grid node. The node is part of the SIG, but it also can provide services independently. There're two entries to the node (Luo et al., 2006):

1. A portal implemented by JSP. User can visit it through Internet browses, such as Internet Explorer and others.

2. A portal implemented by web service and workflow technology. It is special for SIG. Other Grid resources and Grid systems can integrate with our node through this portal.

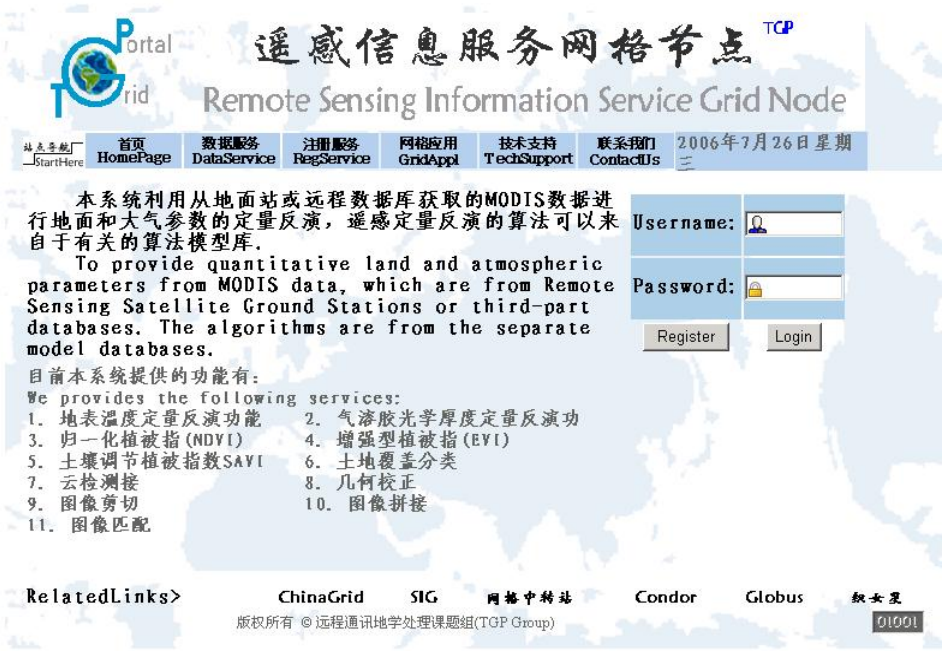

Fig. 1. RSIN web page (URL: http:/www.tgp.ac.cn)

\subsection{Grid Implementation of AOT Retrieval}

Generally speaking, the retrieval process consists of data preparing, data preprocessing, AOT retrieval and post-processing of results. So the Grid service of the SYNTAM AOT retrieval is partitioned into four sub services correspondingly. These sub services are implemented one by one. Upon receiving the user's order of the AOT retrieval service via grid portal, the grid manager initialized an AOT retrieval service and run the data searching sub service to find the MODIS data among the data resource in the Grid pool. Then the data query results are sent to the data preprocessing service. The pre-process service sends the pre-process job to the computing resource and then collect the returned results. The pre-processed MODIS data finally is transferred to the SYNTAM processing service. After processing among computing resource on RSIN, the RSIN collects all of the retrieved AOT 
results and then post-processes them. The final results are sent to the user via the Grid portal. At the same time, the job status is simultaneously monitored by a grid pool manager.

In the following paragraphs, data query schemes, data pre-processing, job management and AOT post-processing are discussed in detail.

\subsubsection{Data Query}

The MODIS data are distributed on RSIN, whose metadata describe the information of the MODIS data, which includes range, producer, quality, date and time, processing methods, satellite, and so on. The data searching service search the registered metadata based on SQL and find out where the required data hosted in. Then query results then returned to the data pre-processing service.

\subsubsection{Data Pre-processing}

Before going to SYNTAM AOT retrieval process, geometrical corrections (including calibration, geo-reference, merging and clipping etc) and radio-metrical calibration should be performed. After that, 16 input parameters required in SYNTAM are in ASCII format and poised to be input to the next phase.

Geo-referencing of MODIS data is time consumable and computationally intensive. Combined with the calibration, the geo-reference task is submitted to the Grid. The algorithms and the partition strategy can be found in the paper from $\mathrm{Hu}$ et al. (2005).

The merging, clipping and format transfer are combined to a unity one. When it concerns to regional or global scale, the partition strategy must be considered if there are no high-powered computer that could handle the merging process in the Grid pool. We apply dynamic filling methods to fulfill the task. Firstly, the request range is divided into regular pieces according to the available computers' amount. The sub range information and the geo-referenced data are sent to the job nodes in the grid environment. Secondly, the job nodes search the data within the specified range and fill the data into the correct location. After the required 16 parameter files are ready on the job node, the SYNTAM AOT services start up.

\subsubsection{Job Management and Post-processing}

The task is partitioned into many sub jobs, which are identified by unique Grid job identifiers. The job manager monitors the job status (running or idle) during the process. Finished status is reached whenever user retrieves all the output files produced by a job. The job is check-pointed for later restart. When a chosen resource refuses to accept the job, the job is vacated and waiting for the manager to reallocate to other computing element. The grid manager collects all the results returned from the job nodes. The results are merged dynamically. After all of the results are merged, the merged files are transformed to the format, which the user required and then the transformed files are finally transferred to the user.

\section{Experiments and Results}

MODIS level 1B data for Europe covering north France and part of England, Netherlands and China, acquired from NASA on July 2, 2006, and November 3, 2004, respectively, are input to RSIN. 
In respect of the AOT retrieval in Europe, Figure 2 shows the preliminary results. The retrieval results are tested with collocated Aeronet in-situ data in Western Europe where 4 Aeronet sites can be available. Figure 3 shows that AOT measurements in Oostende Aeronet site is compared with the collocated MODIS measurements averaging over the area within $5 \mathrm{~km}$ around Oostende. It shows good agreements between SYNTAM retrieval and MODIS Aeronet measurements. The average difference between SYNTAM retrieval AOT value and Aeronet measurements is 0.0165 .
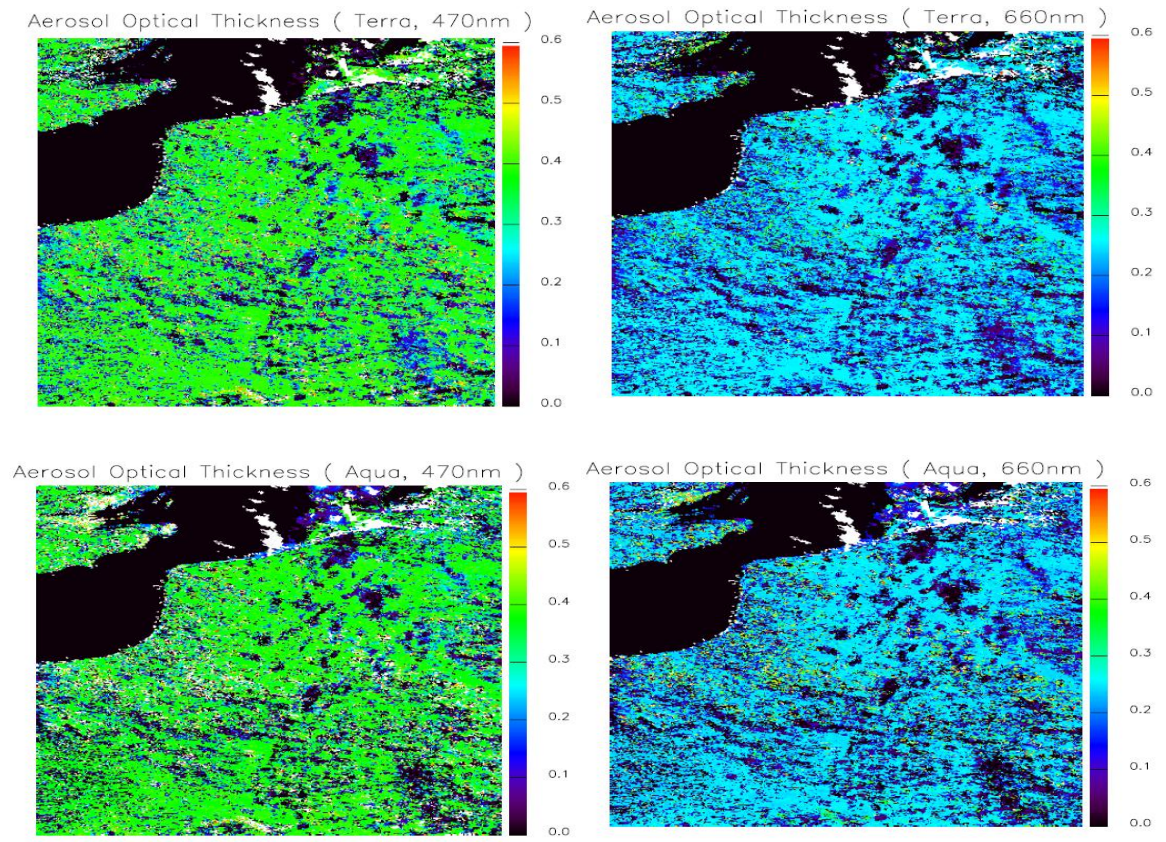

Fig. 2. AOT retrieval from MODIS data on RSIN for a part of Europe on July 2, 2006

\section{Conclusions}

The implementation of the AOT retrieval on RSIN is discussed and tested. In order to check for consistent AOD retrievals, validation for 4 AERONET sites has been incorporated. It is shown in the few examples in this paper that the retrieved AOD compares favorably with the collocated AERONET sun-photometer measurements. Our tests are based on RSIN. The experiments are successful but there are some aspects we should improve in the future. For example, the load balance on RSIN is to be improved. Moreover, the partition strategy doesn't consider the difference among the computing elements. When the job is submitted to node with lower computing ability, the whole efficiency will be affected. Otherwise, when there are high power computers in the grid pool, it may be more efficient to submit most of jobs to them. A scheme should be added in order that able person should do more work. Another 
aspect we should improve is the data management. In our experiments, the database is file based. When the data are centralized in one node, the transferring way will be jam-packed In the future the distributed database should be build with the dynamic replica scheme to reduce the pressure on the data source nodes.

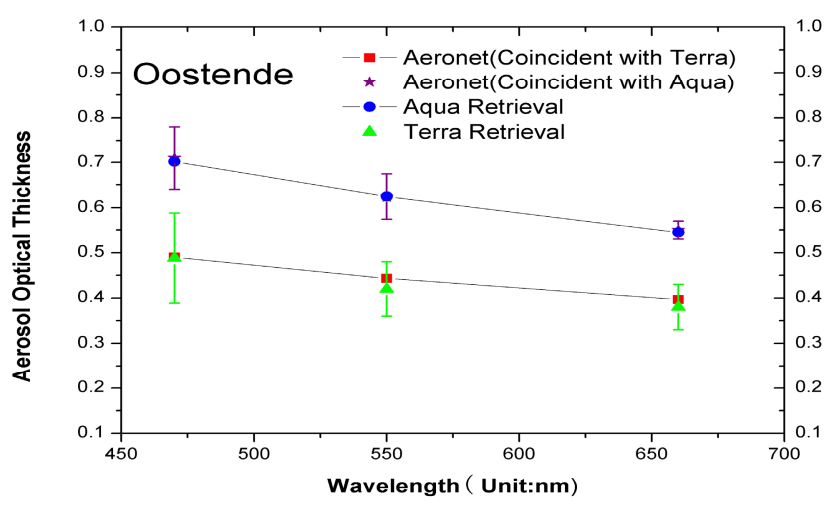

Fig. 3. AOT in Oostende (N 51'13'30", E $\left.02^{\circ} 55^{\prime} 30^{\prime \prime}\right)$, as a function of wavelength

\section{Acknowledgement}

This publication is an output from "Multi-scale Aerosol Optical Thickness Quantitative Retrieval from Remotely Sensing Data at Urban Area" (40671142) and "Aerosol fast monitoring modelling using MODIS data and middlewares development" (40471091) funded by NSFC, China. We are grateful to the MODIS team for making available data used here. Many thanks go to the PI investigators of the mentioned AERONET sites used in this paper and Sina-EU Dragon program.

\section{References}

1. Aloisio, G., Cafaro, M.: A dynamic Earth observation system. Parallel Computing (2003) 1357-1362

2. Aloisio, G., Cafaro, M., Epicoco, I., Quarta, G.: A problem solving environment for remote sensing data processing. In Proceeding of ITCC 2004: International Conference on Information Technology: Coding and Computing held in Las Vegas, NV, USA on 5-7 April 2004,Vol.2. 56-61

3. Cannataro, M.: Clusters and grids for distributed and parallel knowledge discovery. Lecture Notes in Computer Science (2000) Vol. 1823, 708-716

4. Guo Jianping, Yong Xue, Chunxiang Cao, Wuchun Cao, Xiaowen Li, Jianqin Wang, Liqun Fang: eMicrob: a Grid-based Spatial Epidemiology Application. Lecture Notes in Computer Science (2005) Vol. 3516, 472-475

5. Hu Yincui, Xue Yong, Tang Jiakui, Zhong Shaobo, Cai Guoyin: Data-parallel Georeference of MODIS Level 1B Data Using Grid Computing. Lecture Notes in Computer Science (2005) Vol. 3516, 883-886 
6. Intergovernmental Panel on Climate Change 2001-The Scientific Basis (contribution of working group I to the Third Assessment Report of the Intergovernmental Panel on Climate Change). Cambridge University Press, Cambridge (2001)

7. Jacovides, C.P., Varotsos, C., Kaltsounides, N.A., Petrakis, M., Lalas, D.P.: Atmospheric turbidity parameters in the highly polluted site of Athens basin. Renewable Energy (1994) 4 (5), 465-470

8. Kaufman, Y.J., Tanré, D., Remer, L.A., Vermote, E., Chu, A., and Holben, B.N.: Remote sensing of tropospheric aerosol from EOS-MODIS over the land using dark targets and dynamic aerosol models. J. Geophys. Res. (1997) Vol.102, 17051-17067

9. Luo Ying, Yong Xue, Yincui Hu, Chaolin Wu, Guoyin Cai, Lei Zheng,Jianping Guo, Wei Wan, Shaobo Zhong: Remote Sensing Information Processing Grid Node with LooseCoupling Parallel Structure. Lecture Notes in Computer Science (2006) Vol. 3991, 876-879

10. Luo Ying, Yong Xue, Chaolin Wu, Yincui Hu, Jianping Guo, Wei Wan, Lei Zheng, Guoyin Cai, Shaobo Zhong, Zhengfang Wang: A Remote Sensing Application Workflow and Its Implementation in Remote Sensing Service Grid Node. Lecture Notes in Computer Science (2006), Vol. 3991, 292 -299

11. Running, S.W., Justice, C.O., Salomonson, V.V., Hall, D., Barker, J., Kaufman, Y.J., Strahler, A.H., Huete, A.R., Muller, J.P., Vanderbilt,V., Wan, Z.M., Teillet, P., Carneggie, D.: Terrestrial remote sensing science and algorithms planned for EOS/MODIS. International Journal of Remote Sensing (1994), 15(17), 3587- 3620

12. Tang Jiakui, Xue Yong, Yu Tong, Guan Yanning: AOT Determination by Exploiting the Synergy of TERRA and AQUA MODIS (SYNTAM). Remote Sensing of Environment (2005), 94 (3), 327-334

13. Varotsos, C.A., Ondov J. M., Cracknell A. P., Efstathiou, M.N., Long-range persistence in global Aerosol Index dynamics. International Journal of Remote Sensing (2006), 27 (16), 3593-3603

14. Xue, Y., Cracknell, A.P.: Operational Bi-Angle Approach to Retrieve the Earth Surface Albedo from AVHRR data in the Visible Band. International Journal of Remote Sensing (1995), 16(3), 417-429 